\title{
Il diagramma storico-letterario di De Sanctis e la costruzione della identità italiana ${ }^{1}$
}

\author{
María de las Nieves Muñiz Muñiz \\ Universitat de Barcelona \\ muniz@ub.edu
}

\begin{abstract}
La Storia della letteratura italiana di Francesco de Sanctis (1870) viene qui analizzata come segno delle contraddizioni inerenti al processo di costruzione identitaria italiana, una difficoltà di cui sono riflesso le aporie riscontrabili nel suo diagramma.
\end{abstract}

Parole chiave: De Sanctis; storia letteraria italiana; identità italiana.

\begin{abstract}
The Storia della letteratura italiana by Francesco de Sanctis (1870) is analysed as a sign of the contradictions inherent to the construction process of an Italian identity, a difficulty which reflects itself in the aporias to be found in its diagram.
\end{abstract}

Keywords: De Sanctis; Italian literary history; Italian identity.

Che il capolavoro desanctisiano segni in Italia l'atto di nascita della moderna storiografia letteraria, è assioma difficilmente discutibile. Giovanni Getto vi dedicò un noto libro ${ }^{2}$ che ridusse le compilazioni precedenti a mancati abbozzi del modello, e ne fissò i punti cardinali come altrettanti capisaldi per le storie avvenire: narrazione articolata in un continuum progressivo; complesse mediazioni fra il particolare, filologicamente accertato, e il generale organizzato in sistema; specificità del fatto letterario pur nei legami profondi con la vita sociale e con lo sviluppo del pensiero; attenzione all'individualità dei

1. Queste pagine, nate in occasione delle «Giornate di studio su Francesco De Sanctis», organizzate da Rossend Arqués all'Universitat Autònoma di Barcellona (9-11 dicembre 2008), sono state anticipate nel volume di studi Per Romano Luperini, edito a cura di Pietro Cataldi, Palermo: Palumbo, 2010, p. 169-180. Le ripubblico ora in versione ampliata su richiesta del collega Arqués.

2. Giovanni GetTO, Storia delle storie letterarie [1942], nuova edizione riveduta, Firenze: Sansoni, 1969. 
grandi scrittori, visti però come espressione delle tendenze più rappresentative dell'epoca.

Qui vorrei invece rivolgere l'interesse ad altri versanti del problema, e in particolare ai segni lasciati sull'opera dal dibattito che l'aveva preceduta, indicando alcuni anelli deboli della catena che portò ad amalgamare l'identità nazionale italiana con il collante del patrimonio letterario.

Quando nel 1870 vide la luce la Storia di De Sanctis, era ancora incerta la scelta degli autori italiani più emblematici e oscillava il diagramma del loro disporsi per epoche. Il modello bembiano — crescita lineare verso la perfezione rappresentata da Petrarca e Boccaccio e sua clonazione in uno spazio acronico- aveva ricevuto qualche correttivo con i «crescimenti» e «scemamenti» dell'Arcadia, ${ }^{3}$ anche se il diagramma altalenante aveva come punto fisso il perfetto «bello» ("quando ... si arrivi a quella perfezion che costituisce il bello, il volere avanzarsi oltre è il medesimo che dare addietro", scriveva Tiraboschi) $;{ }^{4}$ quanto alla teoria settecentesca del clima, nel primo Ottocento si era allacciata ambiguamente alla linea sociopolitica di Schlegel-SismondiStaël, col risultato di situare l'Italia all'incrocio fra immobilità genetica (il bel paese condannato a contemplare il proprio cielo) e sventura politica (il popolo inerme, asservito e diviso). La scossa più forte, anzi, il capovolgimento dello schema, risaliva alla filosofia vichiana della storia, le cui tesi primitivistiche erano state poi sviluppate da Rousseau e da Herder spingendo a fissare l'autenticità poetica nelle origini -Omero o Dante- e a tracciare una lunga parabola di declino e di rinascita.

Punto in comune tra i vari filoni era la preoccupazione per la decadenza presente e i mezzi per superarla: un fatto imposto dalla necessità di rispondere alle critiche che dal tardo Seicento piovevano sull'Italia (basti ricordare la polemica Bouhours-Orsi $)^{5}$, e il cui flusso era continuato senza soste fino al dibattito tra classicisti e romantici che Madame de Staël aveva suscitato nel 1816. ${ }^{6}$ Le autoapologie imbastite al riguardo e le loro repliche avevano agita-

3. L'Istoria della volgar poesia scritta da Giovanni Mario de' Crescimbeni detto tra gli Arcadi Alfesibeo Cario custode dell'Arcadia, Stamperia di Luca Antonio Chracas, Roma 1698.

4. Storia della letteratura italiana di Girolamo Tiraboschi della Compagnia di Gesù bibliotecario del serenissimo Duca di Modena, Società Tipografica, Modena 1772-1795, t. V, libro I, capo IV.

5. Com'è noto Dominique BouHours, nei suoi Entretiens d'Ariste et d'Eugène (1671), aveva affermato la superiorità della lingua francese e ridotto la poesia italiana a vuoti e malinconici sospiri, mentre nel successivo Manière de bien penser dans les ouvrages d'esprit (1687) criticò le vacue sottigliezze della letteratura più recente. Nel 1703 l'abate Giambattista Orsi rispose alle accuse ricevendo l'appoggio di Muratori, cfr. Corrado VIOLA, Tradizioni letterarie a confronto: Italia e Francia nella polemica Orsi-Bouhours, Verona: Fiorni, 2001.

6. Arcinoto è il dibattito suscitato dall'articolo di Germaine DE STAËL, Sulla maniera e la utilità delle traduzioni, apparso sulla Biblioteca Italiana nel gennaio 1816 (p. 9-18), il cui passo più cocente diceva: «gl'intelletti della bella Italia, se amano di non giacere oziosi, rivolgano spesso l'attenzione di là dall'Alpi, non dico per vestire le fogge straniere, ma per conoscerle; non per diventare imitatori, ma per uscire di quelle usanze viete, le quali durano nella letteratura come nelle compagnie i complimenti, a pregiudizio della naturale schiettezza». 
to il verso e il rovescio dei caratteri della letteratura patria — classicità, suavitas, raffinatezza formale...-, interpretandoli a seconda dei casi come prova di un primato universale o come causa ultima della sua perdita. ${ }^{7}$ Il dilemma tra identità nativa e moderno progresso fu non a caso la croce del Risorgimento, i cui precursori si erano affannati a convertire il marchio di nascita in mito fondativo. Vico era apparso a tale fine lo strumento più adatto, e Foscolo lo aveva adoperato all'altezza del 1806 tracciando nei Sepolcri una curva di ritorno fra Dante e Parini, con in mezzo la schiera di altri grandi: Petrarca, Machiavelli, Michelangelo, Galilei. Vero è che dal sogno si era svegliato presto Leopardi, convinto, già nel 1820, del carattere irreversibile della mutazione storica (qualcosa di più grave e profondo della decadenza):

Da questo stato di corruzione - scriveva in un appunto del dicembre di quell'anno-, l'esperienza prova che l'uomo non può tornare indietro senza un miracolo: lo prova anche la ragione, perchè quello che si è imparato non si dimentica. ${ }^{8}$

Di qui il suo rifiuto dell'imitazione classicista nonostante il persistere dell'ammirazione per gli antichi:

Che smania è questa dunque di voler fare quello stesso che facevano i nostri avoli, quando noi siamo così mutati? di ripugnare alla natura delle cose? di voler fingere una facoltà che non abbiamo, o abbiamo perduta, cioè l'andamento delle cose ce l'ha renduta infruttuosa e sterile, e inabile a creare? di voler essere Omeri, in tanta diversità di tempi??

Né possiamo dimenticare che in quegli stessi anni Manzoni stroncava i modelli classicisti nella lettera a Cesare d'Azeglio, mentre affidava ai protago-

7. Non posso qui entrare nei particolari degli stereotipi più radicati in proposito. Basterà ricordare come dal muratoriano Della perfetta poesia italiana (1706) agli scritti sparsi di Voltaire, classicità e musicalità erano i tratti universalmente attribuiti nel Settecento alla letteratura italiana, e come, proprio per questo, essa era stata ristretta alla sola poesia ed esclusa dal teatro e dalla prosa: i due generi della modernità.

8. Zibaldone di pensieri, appunto datato 9-15 dicembre 1820, p. 403 dell'autografo (cito dall'ed. critica a cura di Giuseppe Pacella, Milano: Garzanti, 1991).

9. Zib. 727-728, 8 marzo 1821. Inedito era ancora lo Zibaldone ai tempi in cui De Sanctis scriveva la sua Storia, come pure il Discorso sopra lo stato presente dei costumi degli Italiani, composto quasi certamente nel 1824, dove Leopardi arrivava a riconoscere un capovolgimento epocale a favore della moderna civiltà, mentre l'Italia appariva sommersa nel proprio letargo, parimenti lontana dall'antico e dal moderno: «'Italia è, in ordine alla morale, più sprovveduta di fondamenti che forse alcun'altra nazione europea e civile, perocché manca di quelli che ha fatti nascere ed ora conferma ogni dì più co' suoi progressi la civiltà medesima, ed ha perduti quelli che il progresso della civiltà e dei lumi ha distrutti. [...] // Sembra che il tempo del settentrione sia venuto. Finora ha sempre brillato e potuto nel mondo il mezzogiorno. Ed esso era veramente fatto per brillare e prepotere in tempi quali furono gli antichi. E il settentrione viceversa è propriamente fatto per tenere il disopra ne' tempi della natura de' moderni", cfr. G. LEOPARDI, Tutte le poesie, tutte le prose e lo Zibaldone, a cura di Lucio Felici e Emanuele Trevi, Roma: Newton Compton Editori, 2010², p. 1020 e p. 1024. 
nisti del suo romanzo una rifondazione ex novo dell'identità italiana. Ex novo perché, scegliendo come eroi due contadini credenti e analfabeti, li spogliava di miti culturali e li rendeva edotti del solo buon senso cristiano. Il passato veniva così raso al suolo, anzi ridotto a una catena di errori culminati nel servile Seicento, "rozzo insieme e affettato», ${ }^{10}$ secondo un ossimoro che risaliva, come vedremo, a Sismondi.

Vico, Muratori, Sismondi: ecco i modelli che si andavano alternando o intrecciando, vuoi per ripristinare le glorie passate, vuoi per farne tabula rasa; ora intendendo l'antico come classico, ora come omericamente primitivo, ora come remora da cui liberarsi.

Non stupisce che le proposte di periodizzazione letteraria susseguitesi tra Sette e Ottocento mostrassero incertezze e discordanze. Così, le prime ad affacciarsi si erano dichiarate concordi nella lode di Petrarca e del Cinquecento, e salvo eccezioni, nella condanna del Seicento, ma si erano divise sul XV secolo (fase di caduta per Crescimbeni, di risalita per Tiraboschi); ${ }^{11}$ mentre quelle successive avevano confermato il ripudio del Barocco, ma avevano allargato i dubbi al Rinascimento. Fondamentale fu al riguardo la tesi di Sismondi secondo cui l'età di Carlo $\mathrm{V}$ aveva posto le basi del futuro declino quale «inevitable effet de l'esclavage de l'Italie». ${ }^{12}$ L'epoca più prestigiosa delle lettere e delle arti italiane era così degradata nel De la littérature du Midi de l'Europe a un'arma a doppio taglio - i fasti dell'impero e il culto della bella forma - individuandovi il germe maligno che doveva svilupparsi nel Seicento sotto specie dell'ossimoro caro a Manzoni: la vuota affettazione, «le faux esprit, la prétention, l'enflure»:

Ainsi le règne de Charles-Quint, malgré toute la gloire qui semble lui être attachée, fut une époque non moins funeste pour l'Espagne que pour l'Italie. [...] Mais, comme nous l'avons déjà pu observer en Italie, ce n'est point au

10. Così nell' Introduzione, a proposito dello stile dell'anonimo secentista. Nella lettera a Fauriel del 29 maggio 1822, lo scrittore applicava la formula a tutta l'epoca: «Sachez donc que je suis enfoncé dans mon roman, dont le sujet est placé en Lombardie, et l'époque de 1628. à 31. Les memoires qui nous restent de cette époque présentent et font supposer une situation de la société fort extraordinaire: le gouvernement le plus arbitraire combiné avec l'anarchie féodale et l'anarchie populaire: une législation étonnante par ce qu'elle prescrit, et par ce qu'elle fait déviner, ou qu'elle raconte: une ignorance profonde, féroce, et prétentieusen (corsivi miei).

11. "[il secolo decimosesto] non sarebbe stato sì lieto e sì fecondo di dotti ed eleganti scrittori, se le fatiche e gli sforzi di que' che gli aveano preceduti, non avessero spianato loro il cammino, e segnata la via», scriveva Tiraboschi nella Premessa al Tomo VI della Storia della letteratura italiana (1776); mentre Crescimbeni sfumava di più il tragitto: "[...] i Lettori resteranno informati dell'origine della nostra Volgar Poesia, e dello stato della medesima, sì appresso gli Antichi come tra i Moderni, e potranno con più agevolezza riconoscere [...] quanto nel primo secolo fosse ella rozza, come nel secondo ingrandisse, come nel terzo cadesse, quanto gloriosamente risorgesse nel quarto, e come varia nel quinto si sia mostrata, infino a i nostri giorni, che a glorioso risorgimento si prepara, mercè dello Studio, e della continua fatica di molti nobilissimi ingegni viventi», cito dall'edizione di Venezia, presso Lorenzo Basegio, 1731, p. 76

12. Jean-Charles-Leonard SiSMONDE DE SisMONDI, Histoire des Republiques Italiennes du Moyen Agge, Zürich: Henri Gessner, 1807, t. I, Introduction. 
moment où une nation perd tous ses avantages politiques, c'est cinquante ans après tout au plus que l'essor de l'esprit s'arrête chez elle, et que sa littérature décline ou finit tout-à-fait. [...] Charles-Quint préparait pour le siècle suivant le faux esprit, la prétention, l'enflure [...]. ${ }^{13}$

Si capisce così che nel 1844 Emiliani-Giudici assegnasse l'originalità al solo Trecento, riscontrando una stasi nel Quattrocento e una perfezione meramente imitativa nel Cinquecento da cui Machiavelli emergeva come unico ingegno libero del secolo. La decadenza veniva dunque allargata ulteriormente in un continuum che includeva l'Arcadia e finiva solo col primo Ottocento grazie ad Alfieri e Foscolo, che, in quanto figli spirituali di Dante, si riagganciavano alle radici originarie:

Ho diviso i nostri annali letterarii in due grandi evi o periodi — chiariva nella Premessa alla Storia delle belle lettere in Italia-. Il primo comprende la letteratura originale; principia dal nascimento della lingua, e finisce con la morte di Lorenzo de' Medici. Il secondo abbraccia la letteratura di perfezionamento e d'imitazione; muove dal Cinquecento, e si chiude con la prima metà del secolo decimonono. ${ }^{14}$

Arrivato il momento di descrivere i periodi, la grande bipartizione si sfumava, tuttavia, in interni chiaroscuri. L'originalità del Trecento, al cui apice campeggiava Dante, appariva attenuata da Petrarca e da Boccaccio malgrado la loro perfezione artistica, o forse in ragion diretta di essa, per la perdita di «vigore» e di «schietta originalità», mentre la stasi del Quattrocento veniva corretta con l'elogio dell'umanesimo, al quale si riconosceva il merito di aver tratto le lettere antiche «dalle tenebre» medievali, anche se aveva interrotto «il corso della letteratura nazionale»:

Lettore, —affermava Emiliani-Giudici nella Lezione X-col cadere del secolo decimoquinto si chiude il primo grande evo storico della italica letteratura; voglio dire il periodo della letteratura originale finisce, e quello della letteratura di imitazione o di perfezionamento incomincia. Nello spazio di circa tre secoli e mezzo lo ingegno italiano nel vortice delle perenni discordie cittadine, dove la Provvidenza lo aveva gettato, godendo la necessaria libertà ad esplicarsi, svolgeva le ragioni tutte di un incivilimento che illuminò la universa Euro-

13. De la littérature du Midi de l'Europe [1813], Paris: Treuttel et Wurtz, 1829, p. 278. Concordi furono invece Muratori e Sismondi nella condanna delle tenebri medievali, seppure l'uno la espresse nel nome del classicismo, l'altro in quello della virtus repubblicana. Nella sua Histoire littéraire d'Italie (Paris: Michaud, 1811-1819), Ginguené tentò una via di mezzo fra le due posizioni, evitando lo stacco fra Quattro e Cinquecento: «Là, se présente à nous un grand spectacle, celui de l'esprit de l'homme se préparant à secouer ses chaînes et reprenant peu à peu sa vigueur, jusqu'à ce que, par un élan que ces premiers efforts avaient préparé, mais qu'ils ne pouvaient faire prévoir, il se relève tout à coup dans le quatorzième siècle à toute sa hauteur, et recommence à briller de tout son éclat» (Préface).

14. Paolo Emiliani-Giudici, Storia delle belle lettere in Italia [Firenze: Società Editrice Fiorentina, 1844], premessa del 1855 alla $2^{\text {a }}$ ed. rivista (cito dalla $4^{\mathrm{a}}$ ristampa di Le Monnier 1865, p. IV). 
pa e sarà sempre celeberrimo nella storia della umanità. Questo portentoso periodo va partito in quattro epoche [...] nella terza epoca sorge Dante Alighieri che sviluppa tutte le capacità della poesia inalzandola fin dove era dato ad ingegno mortale condurla, ne rafferma per sempre le sorti, le immedesima nel principio vitale della nazione, e le esprime col più grande monumento poetico del nuovo incivilimento, con perpetuità d'influenza sopra le arti tutte non che sopra la intera letteratura.

La quarta è l'epoca del Petrarca e del Boccaccio, mercé i quali l'arte, mentre conduce la forma ad altissimo grado, sente il desiderio di fare rivivere l'arte antica e giovarsene; e a fine di emularne legione, si apre nuovi sentieri, vi si spinge animosa, cresce e lussureggia non senza detrimento del proprio vigore e della ingenita e schietta originalità propria. La voglia di satisfare a celesta curiosità, a potestà sublime ambizione, interrompe, nel quattrocento, il corso della letteratura nazionale, ma riesce nello intento di trarre dalle tenebre e risuscitare le lettere antiche, finché il culto della lingua nuova viene ripristinato, trionfa e si diffonde per tutta la penisola. ${ }^{15}$

L'unica figura luminosa restava, dunque, Dante Alighieri, sintesi universale che univa la grandezza letteraria e il vigore civile. Indietro era rimasta la stroncatura del poeta pronunciata da Saverio Bettinelli, ma la luce del grande padre gettava ombra sulle altre due corone, come il primato del Trecento oscurava l'epoca rinascimentale.

Il disagio emerse comunque anche in altri critici di fronte a figure che apparivano anfibie, rendendo anche problematici i loro accostamenti: così, se Sismondi aveva associato il Dante dell' Inferno a un Tasso letto in chiave di Sturm und Drang, Carlo Tenca discordò da Emiliani-Giudici nella scelta monografica di Dante, e vi aggiunse Ariosto col suo sorriso dissolvitore della mitologia pagana, insieme a Tasso, col suo ricupero della tradizione cavalleresca. ${ }^{16}$ Nel tentativo di salvare tutto e tutti a dimostrazione del "primato" italiano, Gioberti propose infine la coppia Dante-Ariosto come superamento della "dualità del reale e dell'ideale», 17 ma poi segnò le distanze fra il pagano Furioso e il "divino» poema ("quasi la Genesi universale delle lettere e arti cristiane»), piegando il tragitto intermedio in una curva declinante:

Se il Furioso si riscontra colla Divina Commedia, dà meraviglia il vedere quanto sia grande l'intervallo morale, che parte questi due poemi; ma se in vece si ragguaglia colle lettere coetanee, (tranne gli scritti del Savonarola), non ci si trova alcun divario notabile rispetto agli spiriti, che li dettarono. Gl'influssi cristiani e cattolici non erano già spenti, ma infievoliti e soverchiati dal risorgente paganesimo. L'Ariosto è in poesia ciò che sono il Caro, il Castiglione, il Casa, il Bembo, il Firenzuola nella prosa amena, il Machiavelli, il Guicciardini, il Giannotti nella politica, e allora o poco appresso il Pomponazzi, il Bruni,

15. Conclusione della Lezione X, ibidem, p. 457-458.

16. Così nell'articolo-recensione sulla Istoria di Emiliani-Giudici, A proposito di una storia della letteratura italiana, Il Crepuscolo, febbraio-marzo, 1852, n. 1.

17. Vincenzo GIOBERTi, Del primato morale e civile degli Italiani, Bruxelles: Meline Cans e C.a, seconda ed. corretta e accresciuta dall'Autore, 1845, p. 382. 
il Sarpi nella filosofia e nella religione. In tutti questi autori l'Idea manca affatto, o di luce abbagliata solamente risplende, perché l'astro viene eclissato dalle ombre interposte della gentilità ricorrente [...]. L'Ariosto, come il Segretario fiorentino, era uomo di cervello troppo robusto e italiano per lasciarsi adescare alla misticità boreale e splenetica dei primi Protestanti; ma non seppe ugualmente cautelarsi contro le profane lusinghe delle lettere antiche, in cui il buono non va scevro dal reo, e contro la sventura de' tempi, nei quali lo splendore dell'Idea cristiana era più che mai annebbiato dai vizi degli uomini, e l'indegna scorza soffocava il midollo.

Queste poche considerazioni bastano a mostrare che la poesia italiana, dall'età di Dante a quella dell'Ariosto, non crebbe, ma andò declinando. ${ }^{18}$

Su quel crinale Tasso si alzava ancora come campione dell'armonia tra classicismo e cattolicesimo, a conferma della cifra nativa della cultura italica («La classica antichità, come italica, è nazionale, e come semiortodossa [...] è affine al cattolicesimo»), ${ }^{19}$ ma il binomio Campanella-Machiavelli riapriva il divario creando un chiaroscuro con più nero che bianco: «due rari ingegni, l'uno dei quali, sagacissimo nello scrutare i cuori e i fatti degli uomini, fece poco caso della giustizia, legittimando i mezzi col fine, e l'altro ideò una utopia così strana, che avrebbe dovuto intitolarla, non dal sole, ma dalla luna». ${ }^{20}$ Non era, insomma, facile armonizzare «il reale e l'ideale», e il diagramma giobertiano si dissolveva in un vago annuncio di palingenesi affidata ai moderni, che senza distinzioni di sorta — da Vico a Balbo-dichiarava eredi di Dante:

Uopo era dunque che la virtù e il nome italiano affatto perissero, o una morale rivoluzione li ritirasse dal letargo, in cui giacevano; e come nel corso della vita organica la specie non si rinnova altrimenti, che mediante il ritorno dell'individualità a' suoi primordii, onde il padre nel figlio rivive e ringiovanisce, così nella storia di un popolo il suo brio morale e intellettivo, non si rinnovella, se non quando rinasce il principio dinamico, che lo produsse. Il quale per le nostre lettere essendo riposto nella Divina Commedia, la risurrezione di Dante era la condizione richiesta pel risorgimento del pensiero e dell'ingegno italiano. Questa risurrezione cominciò in pochi grandi, che figliati intellettualmente dagli scritti dell'uomo sommo, ne rinfrescarono gli esempi, e si stese quindi al resto degli studiosi. E siccome l'Alighieri è moltiforme, universale, e da lui uscì ogni moderna letteratura, come la greca nacque da Omero, ciascuno dei valorosi che calcarono le sue pedate, tolse a studiare ed esprimere in ispecie una parte del suo ingegno: così il Vannetti, il Cesari, il Perticati, applicarono l'animo alla mirabile lingua delle tre Cantiche, il Vico e il Nicolini alla filosofia, Gaspare Gozzi al buon giudizio generalmente, il Varano e il Monti alla poesia, il Giordani allo stile, il Parini all'austerità morale dei pensieri e degli affetti, l'Alfieri, il Foscolo, il Leopardi, il Marchetti, all'idea politica e alla carità patria, il Troya, il Balbo, e altri pochi, alle attinenze colla storia nazionale, il Manzoni alla religione, che pura risplende in ogni parte del

18. Ibidem, p. 393.

19. Ibidem, p. 372.

20. Ibidem, p. 313. 
divino poema. [...] Grazie all'opera di quei valenti, l'ingegno di Dante rivive oggi fra gl'Italiani; e se questa rinascita letteraria, se questa seconda incarnazione di quel divino spirito, fra noi non viene interrotta e soffocata da un nuovo sonno o da straordinaria malignità di fortuna, io non dispero delle sorti civili e religiose della mia patria. $^{21}$

Tali, a volo d'uccello, gli antecedenti della storia che De Sanctis si trovò a riscrivere. Vediamo ora cosa ne risultò, pure in aerea prospettiva. Innanzitutto egli condivise con i predecessori l'idea di Dante come autore della grande sintesi genetica raggiunta nella Commedia (una vasta creazione «dell'artista, del poeta, del filosofo e del cristiano", in cui viveva «involto ancora e nodoso» un mondo che la letteratura moderna avrebbe "realizzato, sottoposto all'analisi, umanizzato", VII: La "Commedia», \$ 46). ${ }^{22}$ Da Foscolo accolse il progetto di una storia letteraria e civile fortemente coesa: «un libro che discerna le vere cause della decadenza dell'utile letteratura, che riponga l'onore italiano più nel merito che nel numero degli scrittori, che si nutra di maschia e spregiudicata filosofia, che col potere dell'eloquenza vi accenda all'emulazione degli uomini grandi» (Dell'origine e dell'ufficio della letteratura, 1809). Ma rimeditò questi principii alla luce di Vico, di Sismondi e di Hegel.

Ne nacquero due diagrammi sovrapposti: l'uno monogenetico e lineare, l'altro più articolato e contraddittorio. La duplicità dei tragitti aveva la sua radice nella concezione ambivalente delle origini: da un lato il "peccato originale» della scuola siciliana e del suo innesto toscano; dall'altro la sintesi incompiuta di Dante (VII $\$ 13$ ).

Il peccato d'origine consisteva nell'aver importato dalla Provenza «concetti e forme» che "non avevano riscontro nella vita nazionale» (Premessa al capitolo I: I Siciliani), come la cultura cavalleresca francese era passata nei cantari senza alcun aggancio con la vita del popolo italiano:

La coltura cavalleresca, se giovò a formare il volgare, impedì la libertà e spontaneità del sentimento popolare, e creò un mondo artificiale e superficiale, fuori della vita, che rese insipidi gl'inizi della nostra letteratura, così interessanti presso altri popoli. Quel contenuto stazionario comincia a moversi presso Guido, di un moto impresso non da sentimento di amore, ma da contemplazione scientifica dell'amore e della bellezza, che se non riscalda il core, sveglia l'immaginazione. Questo dunque si ricordi bene, che la nostra letteratura fu prima inaridita nel suo germe da un mondo poetico cavalleresco, non potuto penetrare nella vita nazionale, e rimaso frivolo e insignificante, e fu poi sviata dalla scienza, che l'allontanò sempre più dalla freschezza e ingenuità del sentimento popolare, e creò una nuova poetica, che non fu senza grande influenza sul suo avvenire. L'arte italiana nasceva non in mezzo al popolo, ma nelle scuole, fra san Tommaso e Aristotele, tra san Bonaventura e Platone (II: I Toscani, \$ 4).

21. Ibidem, p. 394-395.

22. Mi attengo qui e in seguito al testo curato da Niccolò Gallo (Torino: Einaudi, 1958, con introduzione di Natalino Sapegno), limitandomi, per maggiore agilità, a indicare il numero del capitolo e quello dei relativi paragrafi. 
Inoltre lo spirito toscano aveva una tendenza naturale all'eleganza artistica, che, separando forma e contenuto, aveva eroso ulteriormente le basi della non nata letteratura patria:

Questa bella forma, in tanto spirito e vivacità così castigata, propria e semplice e piena di grazia, si andò sviluppando non perché il suo contenuto voleva così, ma in opposizione ad esso contenuto, vuoto ed astratto. Anzi che qualità del contenuto, o di questo e quel poeta, sembra il progresso naturale dello spirito toscano, dotato di un certo senso artistico, che lo tirava alla forma, nella piena indifferenza del contenuto (II: I Toscani, $\$ 2$ ).

Un filo conduttore legava poi il peccato del Duecento a quelli compiuti da Petrarca e da Boccaccio, i quali, in anni di disimpegno civile e di raffinato imborghesimento, avevano aggiunto al purismo formale il culto fine a se stesso dei classici:

Lo studio de' classici, la scoperta di nuovi capilavori, una maggior pulitezza nella superficie della vita, la fine delle lotte politiche col trionfo de' guelfi, la maggior diffusione della coltura sono i tratti caratteristici di questa nuova situazione. La superficie si fa più levigata, il gusto più corretto, sorge la coscienza puramente letteraria, il culto della forma per sé stessa. Gli scrittori non pensarono più a render le loro idee in quella forma più viva e rapida che si offrisse loro innanzi; ma cercarono la bellezza e l'eleganza della forma. Dimesticatisi con Livio, Cicerone, Virgilio, parve loro barbaro il latino di Dante; ebbero in dispregio quei trattati e quelle storie che erano state l'ammirazione della forte generazione scomparsa, e non poterono tollerare il latino degli scolastici e della Bibbia. Intenti più alla forma che al contenuto, poco loro importava la materia, pur che lo stile ritraesse della classica eleganza. Così sorsero $\mathrm{i}$ primi puristi e letterati in Italia, e capi furono Francesco Petrarca e Giovanni Boccaccio (VIII: Il «Canzoniere», $\$ 1$ ).

Boccaccio soprattutto aveva rappresentato la maggior rivoluzione (una vera e propria «catastrofe») con il naturalismo senza ideali del Decameron, che, unito ad altri fattori e al formalismo del Canzoniere, aveva fatto subentrare «all'età virile e credente e appassionata» di Dante, un'altra contraddistinta dall' «l'infiacchimento della coscienza» (IX: $I l$ "Decamerone», $\$ 2$ ).

Vero è che nel diagramma desanctisiano Dante appariva già come una deviazione (salutare) dalla rotta originaria, sicché questo secondo cambiamento di rotta era una deviazione dalla deviazione, e pertanto un ritorno. A complicare le cose, il salto indietro conteneva elementi di progresso e segnatamente, la superiore consapevolezza artistica e una conoscenza più approfondita della cultura classica, acquisti a loro volta indisgiungibili dalla futura decadenza. Il diagramma si piegava così in circolo vizioso mettendo al centro il volto di Giano di Petrarca, arretrato coltivatore dell'arte per l'arte, ma ammirevole maestro di stile:

Ci erano tutti i segni di un grande progresso: una più esatta conoscenza dell'antichità, un gusto più fine e un sentimento artistico più sviluppato, una 
disposizione meno alla fede, che alla critica e all'investigazione, minor violenza di passioni, maggiore eleganza di forme: l'idolo di questa società dovea essere il Petrarca, nel quale riconosceva e incoronava sé stessa. Ma sotto a quel progresso v'era il germe di una incurabile decadenza, l'infiacchimento della coscienza (IX $\$ 3)$.

Anzi, in quanto profondo conoscitore dell'interiorità umana, Petrarca faceva sembrare vecchie le astrattezze dell'allegorismo medievale, e precorreva quella «letteratura moderna» che avrebbe «umanizzato» il mondo della Commedia:

Pare un regresso: pure è un progresso — affermava a proposito del Canzoniere- Questo mondo è più piccolo, è appena un frammento della vasta sintesi dantesca, ma è un frammento divenuto una compiuta e ricca totalità, un mondo pieno, concreto, sviluppato, analizzato, ricerco ne' più intimi recessi. Beatrice sviluppata dal simbolo e dalla scolastica, qui è Laura nella sua chiarezza e personalità di donna; l'amore, scioltosi dalle universe cose entro le quali giaceva inviluppato, qui non è concetto, né simbolo, ma sentimento; e l'amante che occupa sempre la scena, ti dà la storia della sua anima, instancabile esploratore di sé stesso. In questo lavoro analitico-psicologico la realtà pare sull'orizzonte chiara e schietta, sgombra di tutte le nebbie, tra le quali era stata ravvolta. Usciamo infine da' miti, da' simboli, dalle astrattezze teologiche e scolastiche, e siamo in piena luce, nel tempio dell'umana coscienza. Nessuna cosa oramai si pone di mezzo tra l'uomo e noi. La sfinge è scoperta; l'uomo è trovato $($ VIII $\$ 3)$.

Viste così le cose, il diagramma desanctisiano cambia: al ponte lungo tra Dante e i moderni, se ne sovrappone un altro fra l'umanizzazione petrarchesca e il realismo psicologico dell'Ottocento. Non v'è continuità fra questi due tragitti, ma dilemma. Petrarca non nasce da Dante ma contro Dante, proprio perché alla grande sintesi della Commedia mancava la capacità di scendere ad altezza d'uomo, non per difetto d'arte ma per essenza di concezione. Da questo punto di vista la Commedia restava, nel suo migliore «realismo figurale», un'eccezione irripetibile; Petrarca, invece, fungeva meglio da anello di «transizione» perché consentiva di andare per gradi verso l'armonia futura. Tant'è vero che un filo ininterrotto ricongiungeva l'erotica rarefatta del Canzoniere all' «ideale voluptà» di Poliziano e questa al naturalismo idealizzato del Rinascimento:

Il secolo finisce — scriverà De Sanctis a proposito del Quattrocento-, e non hai ancora il libro del secolo, quello che lo riassume e lo comprende ne' suoi tratti sostanziali. Se hassi a dir secolo un'età sviluppata e compiuta in sé in tutte le sue gradazioni, come un individuo, il primo secolo comprende il Dugento e il Trecento, il cui libro fondamentale è la Commedia, e il secondo secolo comincia col Boccaccio ed ha il suo compimento, la sua sintesi, nel Cinquecento. Il Petrarca è la transizione dall'uno all'altro (XI: «Le Stanze», \$17).

Come affrontare in tale prospettiva il problema della decadenza secentesca? I difetti dell'era di Petrarca e Boccaccio erano l'altro anello di congiunzione con l'epoca rinascimentale di cui avevano precorso il vuoto formalismo; 
il Cinquecento a sua volta aveva posto le premesse (qui Sismondi aiutava) del corrotto Seicento. Ma, lungi dal bastare «l'esclavage de l'Italie» a dar conto della crisi, essa appariva ora come fattore accelerante di una lunga evoluzione interna, quella che dal "peccato» originale aveva portato a Petrarca, da Petrarca a Poliziano, da Poliziano alla ambivalenza rinascimentale, corrotta nell'interno, rigogliosa nella forma:

La corruzione e la grandezza del secolo non era merito o colpa di principi o letterati, ma stava nella natura stessa del movimento, ond'era uscito, che ora si rivelava con tanta precisione, generato non da lotte intellettuali e novità di credenze, come fu in altri popoli, ma da una profonda indifferenza religiosa, politica, morale, accompagnata con la diffusione della coltura, il progresso delle forze intellettive e lo sviluppo del senso artistico. Qui è il germe della vita, e qui è il germe della morte; qui è la sua grandezza e la sua debolezza (XII: Il Cinquecento, \$3).

Di qui il dualismo chiastico con il quale De Sanctis dispone le figure di Ariosto e di Machiavelli, il primo creatore nel Furioso di un mondo affascinante per «l'evidenza miracolosa» e per la «serietà artistica», ma vuoto di valori («L'Orlando furioso ti dà la nuova letteratura sotto il suo duplice aspetto, positivo e negativo. È un mondo vuoto di motivi religiosi, patriottici e morali, un mondo puro dell'arte, il cui obbiettivo è realizzare nel campo dell'immaginazione l'ideale della forma», XII $₫ 5$ ); il secondo, profondo conoscitore dell'umana natura anche se affetto da un "materialismo dissimulato» («Il Machiavelli è la coscienza e il pensiero del secolo, la società che guarda in sé e s'interroga, e si conosce; è la negazione più profonda del medio evo, e insieme l'affermazione più chiara de' nuovi tempi; è il materialismo dissimulato come dottrina, e ammesso nel fatto e presente in tutte le sue applicazioni alla vita», XII $\$ 13)$. Quanto a Tasso, il fallimento del suo progetto epico appariva paradossalmente come il trionfo involontario della poesia lirico-elegiaca, segnando così, per un'altra via, la continuità con Petrarca, creatore dell' «idillio italiano" ("L'idillio italiano non è imitazione, ma è creazione originale dello spirito. Già si annunzia nel Petrarca, quale si afferma nel Tasso, un dolce fantasticare tra' mille suoni della natura», XVII: Torquato Tasso, $\$ 12$ ).

La linea dantesca, se emergeva, lo faceva sul versante della "nuova scienza», non della "nuova letteratura»: era il filone politico-filosofico-scientifico di Machiavelli, Bruno, Campanella, Galiei e Sarpi, definiti «formole diverse di uno stesso concetto" (XIX: La Nuova Scienza, \$18).

Le sintesi di reale e di ideale si avverava quindi riaprendo e poi suturando la biforcazione fra scienza e letteratura: prima Vico con la sua grande sintesi del verum e del certum, poi tanti gradini scalati a passo a passo dagli scrittori: Goldoni, col suo realismo limitato, Parini, con «la serietà di un contenuto vivente nella coscienza» (XX: La Nuova Letteratura, $\$ 17$ ); Alfieri, nel quale «l'uomo nuovo» appare ancora «in veste classica»; Foscolo i cui Sepolcri contengono la «profondità di sentire» e l'«affermazione della coscienza rifatta» (XX \$ 20), infine il Romanticismo, che con Manzoni raggiunge la vera "conciliazione» sotto 
le false spoglie della reazione spiritualista (ancora una volta un progresso che sembra un regresso), e approda al «progressivo divenire»:

La base teorica di questa conciliazione è un nuovo concetto della verità, rappresentata non come un assoluto immobile a priori, ma come un divenire ideale, cioè a dire secondo le leggi dell'intelligenza e dello spirito. Onde nasceva l'identità dell'ideale e del reale, dello spirito e della natura, o, come disse Vico, la conversione del vero col certo. Il qual concetto da una parte ridonava ai fatti una importanza che era contrastata da Cartesio in qua, li allogava, li legittimava, li spiritualizzava, dava a quelli un significato e uno scopo, creava la filosofia della storia; d'altra parte realizzava il divino, togliendolo alle strettezze mistiche e ascetiche del soprannaturale, e umanizzandolo. Il concetto adunque era in fondo radicalmente rivoluzionario, in opposizione ricisa col medio evo e con lo scolasticismo, quantunque apparisca una reazione a tutto ciò che di troppo esclusivo e assoluto era nel secolo decimottavo. Sicché quel movimento in apparenza reazionario dovea condurre a un nuovo sviluppo della rivoluzione su di una base più solida e razionale $(\mathrm{XX} \$ 21)$.

Ecco finalmente «l'idea realizzata», la "metafisica» fatta storia (XX $₫ 23$ ). Tutto combaciava: la poesia e la prosa, il linguaggio letterario e quello del popolo. Il tempo della "letteratura nazionale» poteva dirsi giunto dopo aver capovolto il dantesco indiamento del reale in una umanizzazione dello spirito.

Eppure la Storia di De Sanctis si chiude senza riuscire a integrarvi Leopardi: quel poeta-pensatore che aveva messo in dubbio nel 1820 le radici della conciliazione. Non che il suo scetticismo nascesse da freddezza; al contrario, nasceva dalla massima profondità del sentimento. Era, insomma, il sentimento, nel suo più profondo tentativo di conciliarsi col pensiero, a mettere in crisi insieme la «ragione» e l'ideale. L'armonia fra gli opposti si rompeva denudando l'arido vero:

Giacomo Leopardi segna il termine di questo periodo. La metafisica in lotta con la teologia si era esaurita in questo tentativo di conciliazione. La moltiplicità de' sistemi avea tolto credito alla stessa scienza. Sorgeva un nuovo scetticismo che non colpiva più solo la religione o il soprannaturale, colpiva la stessa ragione. La metafisica era tenuta come una succursale della teologia. L'idea sembrava un sostituto della provvidenza. Quelle filosofie della storia, delle religioni, dell'umanità, del dritto avevano aria di costruzioni poetiche. La teoria del progresso o del fato storico nelle sue evoluzioni sembrava una fantasmagoria. L'abuso degli elementi provvidenziali e collettivi conduceva diritto all'onnipotenza dello stato, al centralismo governativo. L'ecletismo pareva una stagnazione intellettuale, un mare morto. L'apoteosi del successo rintuzzava il senso morale, incoraggiava tutte le violenze. Quella conciliazione tra il vecchio ed il nuovo, tollerata pure come temporanea necessità politica, sembrava in fondo una profanazione della scienza, una fiacchezza morale. Il sistema non attecchiva più: cominciava la ribellione. Mancata era la fede nella rivelazione. Mancava ora la fede nella stessa filosofia. Ricompariva il mistero. Il filosofo sapeva quanto il pastore. Di questo mistero fu l'eco Giacomo Leopardi nella solitudine del suo pensiero e del suo dolore. Il suo scetticismo annunzia la 
dissoluzione di questo mondo teologico-metafisico, e inaugura il regno dell'arido vero, del reale (XX $\$ 26)$.

Il filo sciolto di Leopardi spingeva così De Sanctis a riaprire una nuova e più macroscopica duplicità, quella tra il coronamento della sintesi nel segno di Manzoni (approdo ideale della Storia, come capì Debenedetti), ${ }^{23}$ e un incerto avvenire fondato sull'eternizzarsi del paradosso regresso=progresso:

Il secolo sorto con tendenze ontologiche e ideali avea posto esso medesimo il principio della sua dissoluzione: l'idea vivente, calata nel reale. Nel suo cammino il senso del reale si va sempre più sviluppando, e le scienze positive prendono il di sopra, cacciando di nido a tutte le costruzioni ideali e sistematiche [...]. La nuova letteratura, rifatta la coscienza, acquistata una vita interiore, emancipata da involucri classici e romantici, eco della vita contemporanea universale e nazionale, come filosofia, come storia, come arte, come critica, intenta a realizzare sempre più il suo contenuto, si chiama oggi ed è la letteratura moderna [...].

C'è passato sopra Giacomo Leopardi. Diresti che proprio appunto, quando s'è formata l'Italia, si sia sformato il mondo intellettuale e politico da cui è nata. Parrebbe una dissoluzione, se non si disegnasse in modo vago ancora ma visibile un nuovo orizzonte. Una forza instancabile ci sospinge, e, appena quietate certe aspirazioni, si affacciano le altre (Ibidem).

Dante e Petrarca non potevano conciliarsi, allo stesso modo che la lunga marcia di dicotomie e ambivalenze iniziata da quest'ultimo portava di necessità al rinvio ad infinitum della sintesi: il "progressivo divenire», appunto. Il Romanticismo risorgimentale, allacciato al positivismo con l'aiuto di Vico e di Hegel, era stata la chiave di De Sanctis per convertire la palingenesi in storia. La sua onestà consistette nel riconoscere che il problema restava aperto.

$\mathrm{E}$ aperta restava anche (ma forse qui stava il punto) la questione dell'identità nazionale italiana. Perché a ben pensarci, le difficoltà riscontrate da De Sanctis nel tracciare un diagramma lineare, erano inscritte nella indefinitezza stessa delle origini (anzi il tratto originario delle lettere italiane consisteva nell'assenza ab initio di una coscienza e di una realtà nazionale). In fondo non siamo molto lontani dalla visione dantesca dell'Italia come una possibilità negata (o tradita) in origine. Né si discostarono dall'orbita coloro che da Petrarca a Machiavelli, da Alfieri a Foscolo e a Leopardi, videro l'Italia come problema: una possibilità non data, una ricerca, uno stato contraddittorio e inquieto. Non c'è dunque da stupirsi se nell'Ottocento pre e risorgimentale si finì per riporre il carattere italiano in un'ossimorica amalgama di contrasti facendo di necessità - l'assenza di un tratto ben definito- virtù. Ė il filo rosso che congiunge la «dualità del reale e dell'ideale» ricomposta in armonia da Gioberti, alla critica diagnosi di Madame de Staël: «ll y a dans ce pays un bizarre mélan-

23. Cfr. Giacomo Debenedetti, Commemorazione di De Sanctis [1934], poi raccolto in Saggi critici. Nuova serie, Roma: Edizioni del Secolo, 1945, e ora in Saggi, Milano: Mondadori, 1999 («I Meridiani»), p. 387. 
ge de simplicité et de corruption, de dissimulation et de vérité, de bonhomie et de vengeance, de faiblesse et de force» (Corinne ou l'Italie, Livre VI, ch. II). Alla luce di questo problema, la formula manzoniana «un volgo disperso che nome non ha" (coro dell'Adelchi, atto III) acquista significati più inquietanti: essa fissa l'assenza stessa di «nome» — di coscienza e di essere- come premessa genetica della futura identità, condannando gli italiani — come ebbe a dire Massimo d'Azeglio- a rifarsi da capo ${ }^{24}$. Si spiega così meglio che De Sanctis affidasse la soluzione del paradosso al divenire storico come dialettica dei contrari. Ma se l'identità era la storia in quanto avvicinamento infinito all'ideale, ogni sua realizzazione, compresa quella manzoniana, era da considerarsi provvisoria. Oggi pensare a queste cose potrebbe essere utile.

24. Non c'è spazio qui per approfondire il paradosso sottostante all'idea manzoniana di italianità, che, in mancanza di caratteri genetici, poggiò su due puntali: la lingua unita e i precetti del Vangelo assurti a comune e razionale buon senso. 